\title{
Multi-modal myocontrol: testing combined force- and electromyography
}

\author{
Markus Nowak*, Thomas Eiband and Claudio Castellini
}

\begin{abstract}
Myocontrol, that is control of prostheses using bodily signals, has proved in the decades to be a surprisingly hard problem for the scientific community of assistive and rehabilitation robotics. In particular, traditional surface electromyography (sEMG) seems to be no longer enough to guarantee dexterity (i.e., control over several degrees of freedom) and, most importantly, reliability. Multi-modal myocontrol is concerned with the idea of using novel signal gathering techniques as a replacement of, or alongside, sEMG, to provide high-density and diverse signals to improve dexterity and make the control more reliable.

In this paper we present an offline and online assessment of multi-modal sEMG and force myography (FMG) targeted at hand and wrist myocontrol. A total number of twenty sEMG and FMG sensors were used simultaneously, in several combined configurations, to predict opening/closing of the hand and activation of two degrees of freedom of the wrist of ten intact subjects. The analysis was targeted at determining the optimal sensor combination and control parameters; the experimental results indicate that SEMG sensors alone perform worst, yielding a nRMSE of $9.1 \%$, while mixing FMG and SEMG or using FMG only reduces the nRMSE to $5.2-6.6 \%$. To validate these results, we engaged the subject with median performance in an online goal-reaching task. Analysis of this further experiment reveals that the online behaviour is similar to the offline one.
\end{abstract}

\section{INTRODUCTION}

Smooth, natural control of upper-limb prostheses (an instance of myocontrol) is the paradigmatic simple problem which looks simple from an abstract point of view and turns out to be extremely hard in practice. Back in the Fifties surface electromyography (sEMG), formerly a musculoskeletal condition diagnostic technique, began to be used in a two-sensors configuration to open and close a onedegree-of-freedom (DOF) motorized gripper - actually, the first self-powered hand prosthesis in history. Surprisingly, this rudimentary form of control is today still unsurpassed, although (multi-sensor) sEMG was targeted by control theorists and mathematicians soon after the pioneers' era (an early example can be found in [1]).

And yet, proper myocontrol (e.g., control of multi-fingered prosthetic hands) is still by and large unsolved, the main problem being unreliability. On top of this, upper-limb prosthetic hardware is expensive, heavy, badly-looking and clumsy: these are the reasons why self-powered prostheses are so often rejected [2], [3], although better functionality and control are highly desired characteristics of the ideal upper-limb prosthesis in the population of patients [4], [5].

All authors are with the Institute of Robotics and Mechatronics, DLR German Aerospace Center, D-82234 Weßling, Germany.

*Corresponding Author: markus. nowak@dlr. de
Only one commercially available solution is known, namely manufactured by COAPT Engineerings, which employs machine learning. In a word, proper myocontrol is a surprisingly hard problem and twenty-five years of research have not yet produced a reliable, dexterous, natural and clinically accepted system, enabling upper-limb amputees control their prostheses.

Among the ways to improve the situation [3], [6], [7], multi-modal sensing from the human body is an interesting choice. The idea is simple: to gather more information from the surface of the amputee's missing limb than sEMG currently can. This can be enforced by using different kinds of sensors as a substitute, as well as by coupling them to sEMG. Of course one must take into account that the tests used to prove or disprove the validity/feasibility of a technique should resemble as much as possible the real-life conditions in which the interface will be supposed to work [8].

In this paper we focus upon one specific alternative technique, force myography (FMG, [9]), and perform an offline and preliminary online analysis of the optimal combination of FMG and sEMG, with the aim of simultaneous and proportional (s/p) myocontrol of a prosthetic hand and twoDOFs wrist. FMG uses pressure sensors to detect muscle activity through the deformations induced on the stump, contrary to sEMG which detects electrical fields generated by said activity. FMG has potential to detect different information with respect to sEMG [10], provides similar accuracy and better-conditioned signals than SEMG [11], [12] and has already been tested offline and online even on amputees [13]; but as far as we know, a study on the combination of FMG and sEMG is still missing.

Ten intact subjects, fitted with ten sEMG plus ten FMG sensors on the forearm have been engaged in a repetitive trajectory-following task involving the opening/closing of the hand, as well as flexion/extension and pronation/supination of the wrist. Offline, we have tried several sEMG and FMG combinations to predict such muscular activations. Furthermore, the subject who obtained the median offline accuracy was involved in an online version of the experiment, and induced to reach specific target configurations of the hand and wrist. Since the control machine was trained on minimal and maximal values only, whereas the online test involved intermediate activation levels too, the task was far from easy.

If confirmed on a larger scale, our results would indicate that specific combinations of sEMG and FMG sensors could be used in real life to improve $\mathrm{s} / \mathrm{p}$ myocontrol of hand and 

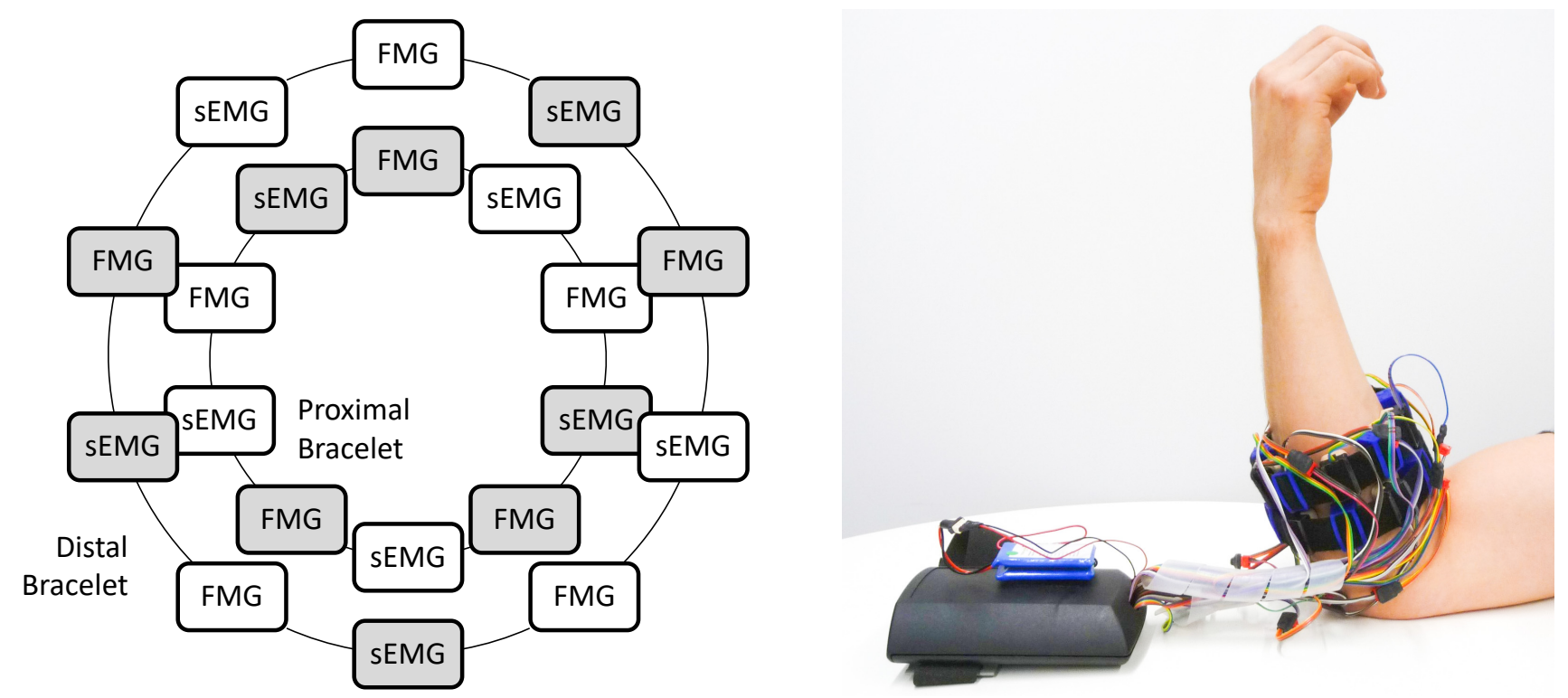

Fig. 1. Left: Schematic showing the arrangement of sensors to be placed on the subjects forearm; Right: image of a subject fitted with the wireless data acquisition device. The sensor are connected by wires to the analog-to-digital converter (in the black box), which then transmits the digital signal via Bluetooth to the PC.

wrist prostheses. This claim is further substantiated by the fact that both the FMG and sEMG sensors we used are small, lightweight and low-power, meaning that they could easily be embedded in a prosthetic socket.

\section{MAterials AND Methods}

The purpose of the study was to investigate the influence of two factors on a combined sEMG and FMG based prosthetic control. Namely these factors are the sensor placement on the proximal end of the users forearm as well as different methods of combining the two sensor types.

We were able to engage ten healthy subjects ( 2 of them female, $26.9 \pm 7.05$ years) in this experiment. All subjects received an oral and written description of the experiment and signed a consent form after all questions were answered. This experiment was approved by the work council of the DLR and conducted in accordance with the Declaration of Helsinki.

Connan et al. [14] developed a wireless data acquisition device for the purpose of investigating multi-modal myocontrol. The initial data analysis in [14] showed promising results for a future use in prosthetic control.

\section{A. Setup}

Similar to [14], we fitted the subjects with two bracelets equipped with sEMG and FMG sensors. However, we arranged the sensors differently. Each bracelet contained both, sEMG and FMG sensors, in alternating order. A schematic of the sensor placement can be found in Fig. 1 on the left hand side and an image of a subject fitted with the device can be found in Fig. 1 on the right hand side.

In total 20 sensors were placed on the forearm, ten sEMG and ten FMG sensors. For further details about the sensors we refer the interested reader to [14].

\section{B. Experimental Protocol}

The participants were asked to perform five different hand and wrist actions, namely

- power grasp,

- wrist flexion,

- wrist extension,

- wrist supination and

- wrist pronation.

These five actions were performed five times each. The users were asked to follow a visual stimulus indicating the respective action.

For our investigations we used a well established regression based machine learning (ML) algorithm, namely Ridge Regression with Random Fourier Features (RRRFF) [15][17]. This ML method represents a finite-dimensional approximation of least squares Support Vector Regression. For details about this approach we refer to the above mentioned publications. However, we would like to mention two of the advantages of this method. RRRFF allows for proportional control and is bounded in space, which means that the computation time for ML training does not depend on the number of samples.

We performed a five-fold cross validation based on the five repetitions mentioned before. To investigate the influence of the placement we only used ten sensors at a time, five sEMG and five FMG sensors. Three different arrangement (or subsets) of sensors were investigated,

- only sensors from the distal bracelet (c1),

- only sensors from the proximal bracelet (c2) and

- an alternating mixture of both bracelets (c3).

Grey boxes in Fig. 1 highlight the third arrangement. All three arrangement cover the full circumference of the forearm. Furthermore, for all three cases the order of sensors 
is alternating. The only difference between those three arrangement is a distal or proximal shift of the sensors. For this analysis we used the so-called stacked approach. This simple sensor mixture model concatenates the two sensors types by simply stacking the signals one after the other in the input vector

$$
\boldsymbol{x}_{\mathrm{st}}=\left[x_{\mathrm{sEMG} 1} \cdots x_{\mathrm{sEMG} 5} x_{\mathrm{FMG} 1} \cdots x_{\mathrm{FMG} 5}\right]^{T} .
$$

The second part of the analysis deals with the comparison of different methods of mixing the two sensors types. For this purpose we compared four different methods, of which two are the two sensor types individually, the third is the above mentioned stacked approach and the forth approach is a hierarchical one, where each sensor type is trained individually and the prediction of these two learning machines serves as the input to a third learning machine combining said outputs. This approach is called ensemble learning [18]. A schematic of these approaches can be found in Fig. 2. For each of the four approaches we used only ten of the 20 sensors in order to have a fair comparison ${ }^{1}$.

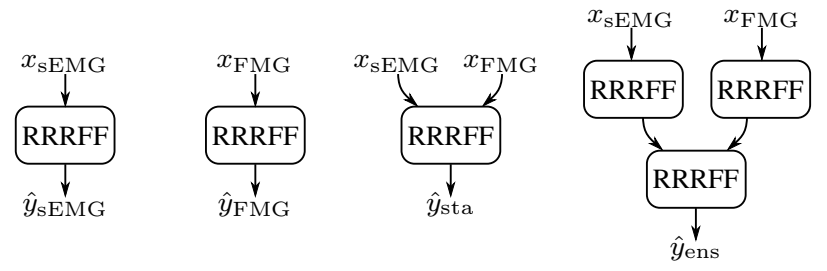

Fig. 2. Schematic representation showing the inputs and outputs of the four sensor type mixing approaches (from left to right: only sEMG, only FMG, stacked, ensemble)

The performance was evaluated using the normalised root mean squared error (nRMSE). All calculations have been performed in MathWorks computing environment MATLAB. Both of the comparisons were statistically evaluated using the open source software environment $R$ [19]. We performed a one-way repeated measures ANOVA with a level of significance of $\alpha=0.05$. As a post-hoc test to determine the individual interactions between the different arrangements and approaches we performed the Tukey-test.

\section{Validation}

Furthermore, we performed an online trial of the sensor mixture comparison to validate our findings. This was a single subject case study. In order to have a fair representation we chose the subject with the median performance in the offline comparison. Said subject was asked to perform a series of online goal-reaching tasks.

Herein the user first performed the same data acquisition session as in the offline case before (five repetitions of five hand and wrist actions), followed by a set of tasks where the user had to reach a certain action based on a visual stimulus. A task was considered successful, when the user could maintain the desired action (within a tolerance of $\pm 15 \%$ per

\footnotetext{
${ }^{1}$ When we used only one sensor type, we processed data from all ten sensors of one type. When we mixed two sensor types, we processed five sensors of each type.
}

$\mathrm{DOF}^{2}$ ) within a certain time (task length: $t_{l}=15 \mathrm{~s}$ ) and for a dedicated time (dwelling time: $t_{d}=1.5 \mathrm{~s}$ ). The performance was evaluated using the success rate $\left(\mathrm{SR}=\frac{\# \text { successful tasks }}{\# \text { all tasks }}\right)$ and the task completion time (TCT), which is the time it took the user to complete a task successfully.

The difficulty here lies in the fact that we only train the ML algorithm on full activation (100\%) of each action and on rest (no activation), while the tasks also require the user to reach intermediate activations of $33 \%$ and $66 \%$, which were not explicitly trained. Since the ML method of choice is a regression based algorithm, interpolation occurs for intermediate values. This way of training a regression method is an instance of a goal-directed on-off set of stimuli, and has already been successfully used in literature [20], [21].

In total the user performed 120 tasks, two repetitions of five different hand/wrist actions at three different activation levels for four different mixing methods.

\section{EXPERIMENTAL RESULTS}

The investigation of the three different placements was evaluated using the nRMSE. Fig. 3 depicts a boxplot of the performance of all ten subjects.

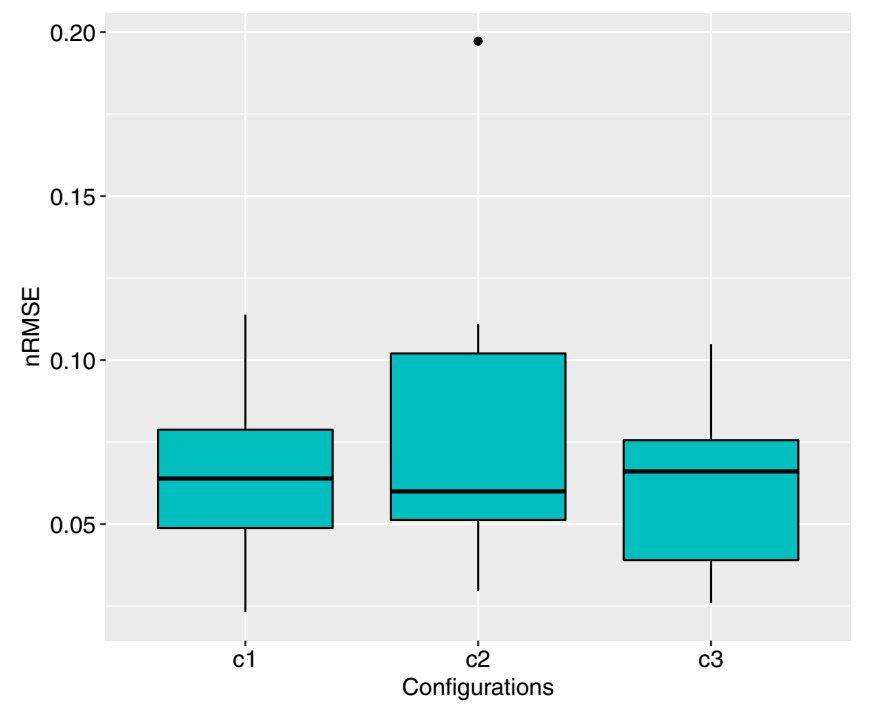

Fig. 3. Boxplot indicating the performance of the machine learning algorithm for three different sensor arrangements based on the data of ten subjects $\left(\mathrm{nRMSE}_{\mathrm{c} 1}=0.0649 \pm 0.0289, \mathrm{nRMSE}_{\mathrm{c} 2}=0.0799 \pm 0.049\right.$ and $\left.\mathrm{nRMSE}_{\mathrm{c} 3}=0.0624 \pm 0.0262\right)$.

A one-way ANOVA was performed to evaluate the results statistically. No significant difference between the three configurations has been found $(F(2,18)=1.33762, p=$ 0.2873). Pairwise interactions have been determined using the Tukey-test, $p=0.397$ for $c 1-c 2, p=0.975$ for $c 1-c 3$ and $p=0.285$ for $c 2-c 3$. For convenience, $c 1$ refers to the proximal bracelet, $c 2$ refers to the distal bracelet and $c 3$ refers to the mixed configuration highlighted in grey in Fig. 1 on the left hand side.

Furthermore, the results of the comparison of the mixing approaches can be found in Fig. 4. Again, we used the

${ }^{2}$ This leads to a target size of less than $1 \%$ of the working space 
nRMSE to evaluate the performance for each of the ten subjects.

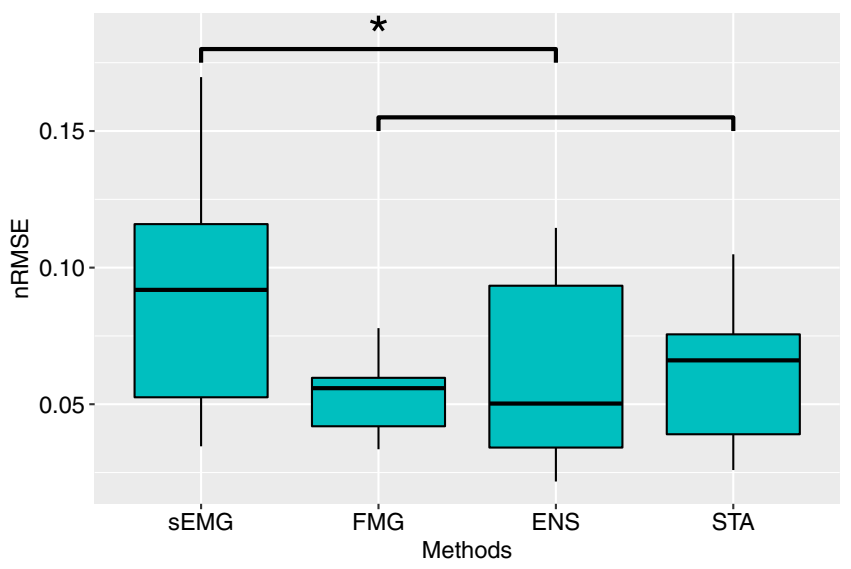

Fig. 4. Boxplot indicating the performance of the mixing approaches based on $s E M G$ only (nRMSE $=0.0912 \pm 0.0434), F M G$ only $(\mathrm{nRMSE}=$ $0.0526 \pm 0.0138)$, ensemble (nRMSE $=0.0575 \pm 0.0314$ ) and stacked (nRMSE $=0.0655 \pm 0.0306$ ) (from left to right)

This comparison was also statistically evaluated using a one-way ANOVA. Significant difference between the four approaches has been found $(F(3,27)=7.05105, p=$ 0.0012). The post-hoc Tukey-test showed that sEMG only is significantly worse than any other of the four approaches with $p$ between $<0.001$ and 0.00784 . The remaining three approaches show no significant difference between one another with $p$ between 0.693 and 0.997 .

To improve the performance of the RRRFF algorithm we performed a hyperparameter $(\sigma)$ optimisation. This was done for each of the three different input signal types, namely sEMG sensors, FMG sensors and for the output of the lower level RRRFF machines in the ensemble approach, see Fig. 2 . The optimal values across subjects are $\sigma_{\text {sEMG }}=0.7$, $\sigma_{\mathrm{FMG}}=0.5$ and $\sigma_{\mathrm{ens}}=0.15$. These values were used in the online case. For the offline analysis the individual optimal values of each subject were used.

The validation involving the median subject of the previous comparison was evaluated using the success rate (SR) and the task completion time (TCT). The result are depicted in Fig. 5.

Since we only engaged one subject in this validation, we didn't perform a statistical evaluation.

\section{DisCUSSION AND CONCLUSIONS}

\section{A. Discussion}

Different sensor configurations yielded no significantly different performances; given this result, we could simplify the experiment (no sensor donning/doffing was required) and acquire data during one session only with 20 sensors, instead of three ${ }^{3}$, keeping unwanted experimental variance to a minimum. As opposed to this, the results of the method comparison are very interesting. A better performance by the

\footnotetext{
${ }^{3}$ Ensemble and stacked use the same sensor configuration.
}

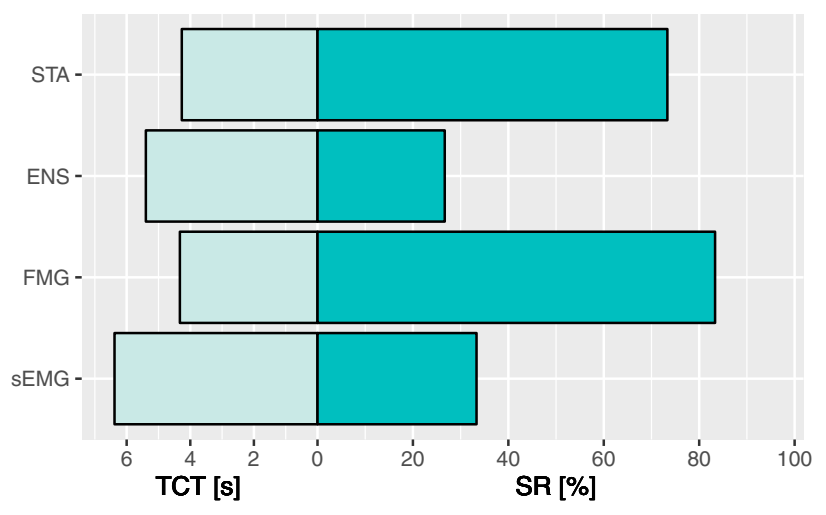

Fig. 5. Results of the online comparison between the four mixing approaches in terms of success rate (SR) on the right hand side and task completion time (TCT) on the left hand side.

multi-modal approach was expected; instead, it is surprising how much better FMG only performed, compared to the state-of-the-art sEMG. The improvement from sEMG only $(\mathrm{nRMSE}=0.0912 \pm 0.0434)$ to $F M G$ only $(\mathrm{nRMSE}=$ $0.0526 \pm 0.0138)$ is significant. A further interesting finding is that the mixture of sensors did not result in an improvement in performance compared to FMG only. The difference between stacked (nRMSE $=0.0655 \pm 0.0306$ ), ensemble $($ nRMSE $=0.0575 \pm 0.0314)$ and $F M G$ only was not significant, see Fig. 4.

The results of the single-subject online validation show a similar behaviour. FMG only shows the best performance (SR of $83.3 \%$ and a TCT of $4.33 \mathrm{~s}$ ) with stacked at a comparable level (SR of $73.33 \%$ and a TCT of $4.27 \mathrm{~s}$ ), while $s E M G$ only shows a rather poor performance (SR of $33.3 \%$ and a TCT of $6.38 s$ ). One particular difference, however, is evident: whereas offline ensemble performed on a similar level as FMG only and stacked, when going online its performance (SR of $26.67 \%$ and a TCT of $5.40 s$ ) is even lower than that of $s E M G$ only. However, we have to keep in mind that these results originate from only one subject and shall therefore serve as an orientation rather than definitive proof.

\section{B. Conclusion}

The two experiments we report about in this paper represent an initial exploration in the direction of improving myocontrol via the combination of different sensor techniques. Fusion of these different sensor information is one of the main ways ahead [8], [12], and FMG alone has already been proved effective in lab-controlled conditions. In this specific case, we have tested several combinations of sEMG/FMG sensors to check whether one can get better results by using them together rather than separately. Their availability, light-weight and low power consumption makes such a combination feasible, at least from the point of view of clinical prosthetics.

We need to remark that the approach was tested online on one subject only; an online test on a wider population is required to better determine whether one sEMG/FMG 
combination, together with a specific machine learning approach, really improves the controllability of the hand/wrist. This is the subject of our immediate future work. Further future research revolves the general issue of $s / p$ control of hand and wrist. In particular, the simultaneous activation of more DOFs, as well as the suppression of the interaction among them must be taken care of. We believe that a tighter integration of sEMG and FMG will bring us closer to an answer to the question.

\section{ACKNOWLEDGMENTS}

We would like to thank Eduardo Ruiz Ramírez for his aid in developing the software basis for the online method comparison and for his initial comparisons between the FMG and sEMG sensors.

\section{REFERENCES}

[1] F. R. Finley and R. W. Wirta, "Myocoder studies of multiple myopotential response," Archives of Physical Medicine and Rehabilitation, vol. 48, no. 11, pp. 598-601, 1967.

[2] B. Peerdeman, D. Boere, H. Witteveen, R. H. in 't Veld, H. Hermens, S. Stramigioli, H. Rietman, P. Veltink, and S. Misra, "Myoelectric forearm prostheses: State of the art from a user-centered perspective," Journal of Rehabilitation Research and Development, vol. 48, no. 6, pp. 719-738, 2011.

[3] A. Fougner, Ø. Stavdahl, P. J. Kyberd, Y. G. Losier, and P. A. Parker, "Control of upper limb prostheses: Terminology and proportional myoelectric control - a review," IEEE Trans Neur Syst Rehab Eng, vol. 20, no. 5, pp. 663-677, 2012.

[4] S. M. Engdahl, B. P. Christie, B. Kelly, A. Davis, C. A. Chestek, and D. H. Gates, "Surveying the interest of individuals with upper limb loss in novel prosthetic control techniques," Journal of NeuroEngineering and Rehabilitation, vol. 12, no. 1, p. 53, 2015. [Online]. Available: http://dx.doi.org/10.1186/s12984-015-0044-2

[5] F. Cordella, A. L. Ciancio, R. Sacchetti, A. Davalli, A. G. Cutti, E. Guglielmelli, and L. Zollo, "Literature review on needs of upper limb prosthesis users," Frontiers in Neuroscience, vol. 10, p. 209, 2016. [Online]. Available: http://journal.frontiersin.org/article/10. 3389/fnins.2016.00209

[6] J. Lobo-Prat, P. N. Kooren, A. H. Stienen, J. L. Herder, B. F. Koopman, and P. H. Veltink, "Non-invasive control interfaces for intention detection in active movement-assistive devices," Journal of NeuroEngineering and Rehabilitation, vol. 11, no. 1, p. 168, 2014. [Online]. Available: http://dx.doi.org/10.1186/1743-0003-11-168

[7] Y. Fang, N. Hettiarachchi, D. Zhou, and H. Liu, "Multi-modal sensing techniques for interfacing hand prostheses: A review," IEEE Sensors Journal, vol. 15, no. 11, pp. 6065-6076, Nov 2015.

[8] N. Jiang, S. Dosen, K.-R. Müller, and D. Farina, "Myoelectric control of artificial limbs - is there a need to change focus?" IEEE Signal Processing Magazine, vol. 29, no. 5, pp. 148-152, 2012.

[9] S. L. Phillips and W. Craelius, "Residual kinetic imaging: A versatile interface for prosthetic control," Robotica, vol. 23, no. 3, pp. 277-282, May 2005. [Online]. Available: http://dx.doi.org/10.1017/ S0263574704001298

[10] V. Ravindra and C. Castellini, "A comparative analysis of three noninvasive human-machine interfaces for the disabled," Frontiers in Neurorobotics, vol. 8, no. 24, 2014.

[11] D. Yungher, M. Wininger, W. Baar, W. Craelius, and A. Threlkeld, "Surface muscle pressure as a means of active and passive behavior of muscles during gait," Medical Engineering \& Physics, vol. 33, pp. 464-471, 2011.

[12] M. Connan, E. Ruiz Ramírez, B. Vodermayer, and C. Castellini, "Assessment of a wearable force- and electromyography device and comparison of the related signals for myocontrol," Frontiers in Neurorobotics, vol. 10, no. 17, 2016.

[13] E. Cho, R. Chen, L.-K. Merhi, Z. Xiao, B. Pousett, and C. Menon, "Force myography to control robotic upper extremity prostheses: A feasibility study," Frontiers in Bioengineering and Biotechnology, vol. 4, p. 18, 2016. [Online]. Available: http: //journal.frontiersin.org/article/10.3389/fbioe.2016.00018
[14] M. Connan, E. Ruiz Ramírez, B. Vodermayer, and C. Castellini, "Assessment of a wearable force- and electromyography device and comparison of the related signals for myocontrol," Frontiers in Neurorobotics, vol. 10, p. 17, 2016. [Online]. Available: http://journal.frontiersin.org/article/10.3389/fnbot.2016.00017

[15] A. Rahimi and B. Recht, "Random features for large-scale kernel machines," in Advances in Neural Information Processing Systems 20, 2008, pp. $1177-1184$.

[16] _ - "Uniform approximation of functions with random bases," in Allerton Conference on Communication Control and Computing (Allerton08), Sep. 2008, pp. 555-561.

[17] A. Gijsberts, R. Bohra, D. S. González, A. Werner, M. Nowak, B. Caputo, M. A. Roa, and C. Castellini, "Stable myoelectric control of a hand prosthesis using non-linear incremental learning," Frontiers in Neurorobotics, vol. 8, no. 8, pp. 1-15, 2014. [Online]. Available: http://www.frontiersin.org/neurorobotics/10.3389/ fnbot.2014.00008/abstract

[18] T. G. Dietterich, "Ensemble learning," The handbook of brain theory and neural networks, vol. 2, pp. 110-125, 2002.

[19] “The R project for statistical computing," https://www.r-project.org/, accessed: 2017-05-12.

[20] A. Gijsberts, R. Bohra, D. Sierra González, A. Werner, M. Nowak, B. Caputo, M. Roa, and C. Castellini, "Stable myoelectric control of a hand prosthesis using non-linear incremental learning," Frontiers in Neurorobotics, vol. 8, no. 8, 2014.

[21] D. Sierra González and C. Castellini, "A realistic implementation of ultrasound imaging as a human-machine interface for upper-limb amputees," Frontiers in Neurorobotics, vol. 7, no. 17, 2013. 